\title{
Canine Cryptorchidism: A Concise Review of its Origin, Diagnosis and Treatment Caroline Spangenberg
}

\author{
Caroline Spangenberg
}

\begin{abstract}
Cryptorchidism is a heritable, autosomal recessive trait in dogs. Due to the anticipated nature of the defect, animals with this condition should not be used for breeding as it poses the possibility of the trait being inherited by offspring. The incidence rate of cryptorchidism in dogs is reported to be ranging from 0.8 to $10 \%$ with a relatively higher prevalence in smaller breeds and purebred dogs, such as the English Bulldog, Boxer, Chihuahua, Shetland Sheepdog, Siberian Husky and Yorkshire Terrier. This report gives a brief overview of origin, diagnosis and treatment of canine cryptorchidism.
\end{abstract}

Key words: Cryptorchidism, Autosomal, Recessive, Breeding

\section{BACKGROUND}

Under normal circumstances, there should be two readily palpable testicles present in the scrotum of a male dog. The term 'cryptorchid' refers to the birth defect that occurs when one or both of the testicles does not descend from the abdomen into the scrotum. ${ }^{[1-6]}$ Unilateral cryptorchidism occurs when only one testis does not descend, while bilateral cryptorchidism occurs when neither testis descends. ${ }^{[5]}$ In the case of a unilateral cryptorchid, only one testicle will have descended while the other remains retained somewhere in the abdomen, in the inguinal canal, or the pre-scrotal area. ${ }^{[5]}$ Cryptorchidism is a heritable, autosomal recessive trait in dogs, meaning that it can be carried by both the dam and the sire and can be passed to the offspring by either of them. ${ }^{[5,7]}$ For this reason, dogs with this defect should not be bred, as it is likely that they could pass it on to their offspring. ${ }^{[5]}$ Among some of the most common congenital defects observed in small animal medicine, cryptorchidism is amongst the most prevalent, with the reported incidence ranging from 0.8 to $10 \%$ and smaller breeds being 2.7 times more likely to develop cryptorchidism than larger breeds. ${ }^{[3]}$ Furthermore, while the incidence of cryptorchidism has been recorded as higher in purebred dogs and is often seen as a pattern within families, there are also certain breeds that have presented a higher incidence and are thus at a greater risk for developing cryptorchidism. ${ }^{[3,5]} \mathrm{A}$ few of these breeds are the English Bulldog, the Boxer, Chihuahua, Shetland Sheepdog, Siberian Husky and Yorkshire Terrier. ${ }^{[3,5]}$ Generally, by the time the animal has reached six months of age, testicular descent should have occurred and the testicles will have reached their final scrotal position. ${ }^{[5,7]}$ It is not until the animal has reached this point in puberty that they are deemed cryptorchid if testicular descent has not yet transpired. Depending on the location of the retained testis, there are a variety of surgical approaches that can be taken to successfully castrate the dog. There is an array of critical complications that can occur as a result of the condition that are dependent upon various factors related to the retained testicle(s), such as age of the animal and the location and degree of severity of the retained testicle..$^{[7,8]}$

\section{Development}

Cryptorchidism develops under a number of biological influences. In order for normal sexual development to occur in the male dog, there needs to be sex chromosomes, normal development of the testicles and normal developmental of the external genitalia under the influence of sex hormones, such as testosterone, luteinizing hormone, follicle stimulating hormone or oestrogen. ${ }^{[7]}$ It is not until after day 30 of gestation that the sex of the embryo becomes distinguishable. ${ }^{[7]}$ In the normal dog, sex cords will be the first structure to develop near the kidneys, which will then differentiate into either the ovaries or testes. ${ }^{[7]}$ Under the influence of the SRY gene, which is present only on the Y chromosome, testicles will develop and the foetus will prove to be a male. ${ }^{[7]}$ There is a substance called the testis determining factor (TDF) that will determine whether there is male or female development and it too is controlled by the $\mathrm{Y}$ chromosome. ${ }^{[7]}$ After day 36 of gestation, if male development has been determined under the influence of these genes and substances, testicular differentiation will

Cite this article : Spangenberg C. Canine Cryptorchidism: A Concise Review of its Origin, Diagnosis and Treatment Caroline Spangenberg. BEMS Reports. 2021;7(1):1-3.

\section{Caroline Spangenberg} UCD School of Veterinary Medicine, University College Dublin, Belfield, Dublin 4, IRELAND.

\section{Correspondence}

Caroline Spangenberg

School of Veterinary Medicine, University College Dublin, Belfield Dublin 4, IRELAND.

Phone no: +353 (83)3784346

E-mail: caroline.spangenberg@ ucdconnect.ie

History

- Submission Date: 05-10-2020;

- Review completed: 14-10-2020;

- Accepted Date: 29-10-2020.

DOI : 10.5530/bems.7.1.1

Article Available online

http://www.bemsreports.org

Copyright

(c) 2021 Phcog.Net. This is an openaccess article distributed under the terms of the Creative Commons Attribution 4.0 International license. 
proceed. ${ }^{[7]}$ After initial development, the testes will lie near the kidneys within the abdominal cavity beneath the peritoneum, with the right testicle being slightly cranial to the left. ${ }^{[1,7]}$ The end of a testicle is attached to a mesenchymal cord called the gubernaculum, which runs through the inguinal canal (the passageway between the deep internal inguinal ring and the superficial external inguinal ring) and attaches to the developing scrotum. ${ }^{[2,7]}$ There is an evagination of the peritoneum in the inguinal region called the vaginal process. ${ }^{[2]}$ It is through the vaginal process that the gubernaculum passes, forming the vaginal cavity. ${ }^{[2]}$ Under the stimulation of certain peptide hormones, the gubernaculum will enlarge in order to apply pressure to the testes to keep them in place during the process of testicular descent. ${ }^{[2,6,7]}$ For normal testicular descent to begin, the presence of testosterone is required to stimulate the development of the genitofemoral nerve, which innervates the gubernaculum and induces its differentiation..$^{[2]}$ As the gubernaculum continues to grow, it will cause an expansion of the inguinal canal that will consequently pull the testis and the epididymis towards the scrotum until the testicles have moved through the inguinal canal and the gubernaculum responds by shrinking while the testes settle into their final position in the scrotum. ${ }^{[2,6,7]}$ As the gubernaculum has its own nerve supply, the genitofemoral nerve, it will be stimulated to shrink and regress following descent of the testicles. ${ }^{[2]}$ The testicles typically pass through the inguinal canal three to four days after birth and reach their final scrotal position by days 14-35 of age. ${ }^{[7]}$ In summary, testicular descent under normal circumstances occurs by the movement of the testes through the abdominal cavity and into the deep inguinal ring, followed by movement through and ultimately out of the inguinal canal where they will then move into their final scrotal position.

\section{Diagnosis}

Diagnosing cryptorchidism as well as locating the retained testicle can be achieved by conducting a physical exam and through visual examination. Careful palpitation of the scrotum and the region of the inguinal canal can be an accurate indicator, but you have to be careful not to mistake fat or a lymph node for a retained testicle, which is quite common. ${ }^{[5,7]}$ If it is a testicle, it should be able to move freely and it should have a palpable epididymis attached to it. ${ }^{[7]}$ One method for locating the retained testis is examination by ultrasonography, but this can be difficult depending on if the testicle is retained in the inguinal canal or in the abdomen. ${ }^{[5,7]}$ If it is in the abdomen, the testicle may be smaller in size as well as it could be in a wider range of areas in the abdominal cavity, thus ultrasonography is typically more successful in locating inguinally retained testicles. ${ }^{[4,7]}$ If you are able to successfully use an ultrasound to locate a testicle retained in the abdomen, it should have a similar structure to a scrotal testis, unless there are complications. ${ }^{[7]}$ On the other hand, if ultrasonography is not successful or the testicle(s) are not palpable, another option is to perform an hCG or GnRH stimulation test. This test will be able to determine if testicular tissue is still present, which would be an obvious indicator that there is a retained testicle. ${ }^{[7]}$ This test is conducted by obtaining a baseline testosterone and then administering a stimulating hormone, such as hCG or GnRH. ${ }^{[5,7]}$ Testosterone concentrations are then obtained at 1and 4 -hr post injection. ${ }^{[7]}$ If the baseline concentration of testosterone has more than doubled, that is a clear indication that testicular tissue is present and the animal is cryptorchid. ${ }^{[7]}$ It is worth mentioning that it has been previously observed that cryptorchid males will tend to have a less obvious response to an hCG or $\mathrm{GnRH}$ stimulation test than those males which have had successful testicular descent. ${ }^{[7]}$ It is also interesting to note that, while it has been considered, there has been no indication that any lack or excess of hormone levels, such as testosterone, luteinizing hormone, follicle stimulating hormone or oestrogen, play a role in cryptorchidism incidence. ${ }^{[7]}$ This has been proven through measuring the levels of these hormones in both normal males and cryptorchid males and observing that there is no obvious difference and thus treatment with a stimulating medication, such as those hormones mentioned above, would not resolve or prevent the condition. ${ }^{[7]}$ Furthermore, there is the occasional incidence of the apprehensive individual who will retract their testes towards their body when touched and in this case, massaging the inguinally retained testes towards the scrotum can be performed. ${ }^{[7]}$

\section{Treatment}

To treat cryptorchidism, it would be advisable to remove both testes surgically. If the animal is a unilateral cryptorchid, one descended testis will still likely be able to produce sperm. ${ }^{[5]}$ On the other hand, a bilaterally cryptorchid animal would be sterile, as the testes need to be 4 to 5 degrees cooler than body temperature in order to produce normal sperm and both testis would be retained in the high temperature of the abdomen, thus killing or impairing the sperm and rendering process of successful spermatogenesis impossible. ${ }^{[5,7]}$ In the case of a unilateral cryptorchid, not removing the descended testis would leave the possibility of passing the defect on to offspring. ${ }^{[7]}$ Furthermore, there are several negative side effects that could occur as a result of the placement of the retained testes.

Testicular descent should generally be completed by the time the animal has reached six months of age. ${ }^{[7]}$ It is recommended to wait up until about six months of age, or until puberty is fully attained, before declaring an animal as cryptorchid. ${ }^{[7]}$ By the time six months have passed after birth, the inguinal rings of most dogs have closed, which should halt movement of the testicles from the abdomen into the inguinal canal and into the scrotum. ${ }^{[7]}$ The testicles typically pass through the inguinal canal three to four days after birth and reach their final scrotal position by days $14-35$ of age. ${ }^{[7]}$ While six months of age is widely accepted as the age an animal can be deemed cryptorchid if they do not yet have two scrotal testicles, there is still plenty of room for individual and breed specific variation. ${ }^{[7]}$ This variation can be attributed to the diversity in puberty stages between breeds. ${ }^{[7]}$ On average, one could expect both testes to be descended by two months of age for any given breed and for any time longer than that to be suspicious of cryptorchidism. ${ }^{[7]}$ For a larger breed, this may not be the case, as testicular descent may not be complete until the animal is over a year old. ${ }^{[7]}$ On the other hand, for smaller and medium sized animals, six months is considered to be the cut-off for testicular descent before the animal is deemed cryptorchid. ${ }^{[8]}$ Despite this information, after considering the breed of the animal, there is really no need to delay surgery any longer than six months of age.There are a variety of surgical approaches that can be taken to successfully castrate the dog, but it is dependent upon the location of the retained testis. ${ }^{[4,5,7]}$ The testicle is likely to be retained abdominally if it began to enlarge before passing into the inguinal ring or if its descent had been slowed by some other factor. ${ }^{[7]}$ If the testicle began to enlarge after it had entered the deep inguinal ring, but before exiting the superficial inguinal ring, or if normal descent through the inguinal canal is altered/does not occur at all, the testicle may become retained in the inguinal region, deeming the animal an inguinal cryptorchid. ${ }^{[7]}$ It is more likely for the right testicle to be retained than the left due to its position in the abdominal cavity, which is slightly more forward. ${ }^{[7]}$ Additionally, location of the retained testicle correlates with the size of the testis, as abdominally retained testicles are smaller in size than the inguinal testes and inguinally retained testes are smaller than testes that have travelled outside of the external inguinal ring but have not yet reached the scrotum. ${ }^{[7]}$ Furthermore, there is a reduction in sperm production in retained testicles, which worsens 
with the degree of retention. ${ }^{[7]}$ In terms of the surgical approach for finding and removing the retained testicle, the location does indeed have an effect on the approach method. In order to find the retained testicle, the ductus deferens must be identified and followed leading to the testicle, which may be abdominally or inguinally retained. ${ }^{[5]}$ If the ductus deferens can be identified as passing through the inguinal ring, the testis is not abdominal. Once the retained testicle has been identified, there are a handful of different incisions that can be made depending on the location. These are discussed below.

In human medicine, treatment of cryptorchidism is performed with both surgery and hormonal therapy ${ }^{[7]}$ Doctors are not concerned with male fertility, as there are many techniques and options available for the sterile human male and it is unlikely for them to pass the trait onto their offspring. ${ }^{[7]}$ A physician's main concern in human medicine is reducing the risk for testicular neoplasia. ${ }^{[7]}$ In veterinary medicine, while reducing the risk of neoplasia is also a great concern, the other major concern is the passing of heritable defects, as the male could still be fertile under certain circumstances. ${ }^{[7]}$ For this reason, castration is the most common choice of treatment for cryptorchidism. ${ }^{[5,7]}$ The retained testicle can be removed laparoscopically or as a laparoscopic-assisted procedure or by performing certain incisions. ${ }^{[4]}$ In the incidence of inguinal or pre-scrotal retained testes, removal will be completed by performing a typical castration incision. ${ }^{[4]}$ If the testis is abdominal, on the other hand and has strayed far from the standard midline incision, the surgical approach will involve incising directly over the retained testicle. ${ }^{[4]}$ A parapreputial skin incision or a paramedian abdominal wall incision are two of the most common incisions that are performed for removal of abdominal testicles. ${ }^{[4]}$ The paramedian abdominal wall incision has often proved to be the less preferred method, as it can be messy due to muscular haemorrhage, as well as it provides limited access to the abdominal cavity. ${ }^{[4]}$ Diversely, a parapreputial skin incision with a midline abdominal wall incision is preferred, as there is decreased haemorrhage and better visualization, albeit a greater degree of tissue dissection is required. ${ }^{[4]}$ This midline incision can be extended to locate the testis if it is not easily identified, as an abdominally retained testicle could be located anywhere between the kidney's and the inguinal ring. ${ }^{[4]}$ Making this extended incision will cause less tissue trauma. Most importantly, it is critical to be sure that the structure that has been located and that will be ligated and removed is indeed the retained testicle and not a different abdominal structure. ${ }^{[4]}$ Lastly, surgical placement of the retained testicle into the scrotum is not recommended and surgery should not be completed in dogs under six months old in the case of late descent. ${ }^{[7]}$

\section{Complications}

There are various complications that can occur as the result of cryptorchidism, including sterility, neoplasia, local pain, behaviour disturbances, skin diseases and testicular torsion resulting in abdominal pain and other complications, to name a few. ${ }^{[8]}$ The development of these complications is dependent on certain determining factors, such as age of the animal and the location and degree of severity of the retained testicle, if that can be measured. ${ }^{[8]}$ Testicular neoplasia can be observed through clinical signs that include swelling and enlargement in the area in which the testicle is retained and occasionally weight loss, lethargy, decreased appetite, or vomiting, but these indicators are not always observed. ${ }^{[8]}$ Often times there are not very obvious clinical signs for testicular tumours, but there is an increased incidence of Sertoli cell tumours in abdominal testes and for that reason, if an abdominal testis is neoplastic, it may be larger or swollen or have an abnormal appearance as compared to the other testis, which should be visible either through ultrasonography or upon removal. ${ }^{[8]}$ Furthermore, there have been incidences recorded when Sertoli cell tumours have led to a condition called hyperestrogenism as a result of the tumour producing oestrogen and causing signs of feminization in the animal. ${ }^{[8]}$ In this case, some of the observable clinical signs could include enlarged mammary glands and nipples, as well as hair loss, hyperpigmentation of the skin, anaemia, lethargy and a reduced sex drive or squatting to urinate. ${ }^{[8]}$ These clinical signs, although, are less commonly observed.

\section{ACKNOWLEDGEMENT}

Many thanks to Dr. David Kilroy and Dr. Arun Kumar of the University College Dublin School of Veterinary Medicine for their support and guidance during the writing of this article.

\section{CONFLICT OF INTEREST}

The author declares no conflict of interest.

\section{ABBREVIATIONS}

SRY gene: The Sex-determining Region Y Gene; TDF: Testis Determining Factor; hCG: Human Chorionic Gonadotropin; GnRH: Gonadotropin-releasing Hormone.

\section{REFERENCES}

1. Evans HE, DeLahunta. Chapter 2 (Prenatal Development) in Miller's Anatomy of the Dog, $4^{\text {th }}$ ed, Elsevier. 2013

2. Arighi M. Testicular Descent and Cryptorchidism. Current Therapy in Equine Reproduction. 2007;185-94. doi:10.1016/b978-0-7216-0252-3.50033-3.

3. Birchard S, Michael N. Cryptorchidism. Vet Folio, Internal Medicine Compendium. 2008. www.vetfolio.com/learn/article/cryptorchidism.

4. "Cryptorchid Castration: Approach. Veterinary Surgery. 2003. www. vetsurgeryonline.com/cryptorchid-castration-approach.

5. England G, Verstegen J. Canine and Feline Cryptorchidism. Recent Advances in Small Animal Reproduction, by P. W. Concannon, International Veterinary Information Service. 2001.

6. Hutson JM, et al. The Role of the Gubernaculum in the Descent and Undescent of the Testis. Therapeutic Advances in Urology. 2009;():115-21. doi:10.1177/1756287209105266.

7. Pinard C. Testicular Tumors. Vca_Corporate. 2017. vcahospitals.com/know-yourpet/testicular-tumors.

8. Lopate C. Cryptorchidism in the Dog: How It Happens, How to Diagnose Whether to Treat. Reproductive Revolutions. www.reproductiverevolutions. com/RR_files/pdf_docs/Cryptorchidism_in_dogs_how_why_and_what_to_do_ about_it.pdf. 